\title{
INFLUÊNCIA DE FATORES ENDÓGENOS NA PERCEPÇÃO ELETROFISIOLÓGICA E COMPORTAMENTAL DE GRAPHOLITA MOLESTA (BUSCK) (LEPIDOPTERA: TORTRICIDAE) A VOLÁTEIS DE PLANTAS HOSPEDEIRAS
}

\section{D.L. Altafini* ${ }^{*}$ J. Sant'Ana, L.R. Redaelli, R. Lorscheiter}

Universidade Federal do Rio Grande do Sul, Faculdade de Agronomia, Av. Bento Gonçalves, 7712, CEP91540000, Porto Alegre, RS, Brasil. E-mail: deisila@gmail.com

\section{RESUMO}

A mariposa-oriental Grapholita molesta (Lep.: Tortricidae) destaca-se como uma das principais pragas das rosáceas no Brasil. Durante a alimentação, as lagartas fazem galerias em brotos e frutos, prejudicando a produção comercial. Este trabalho objetivou estudar a influência de voláteis de plantas de macieira e pessegueiro e a interação destes com o feromônio sexual sintético, na percepção eletrofisiológica e no comportamento quimiotáxico (olfatometria) da espécie. Observou-se a percepção eletroantenográfica de ambos os sexos de G. molesta, alimentados ou não, a extratos de broto, fruto verde e fruto maduro de macieira (Malus domestica, var. Gala) e pessegueiro (Prunus persicae, var. Chiripá). Os fatores endógenos avaliados foram a idade, o status de cópula e a alimentação, em resposta ao extrato de broto de pessegueiro (EBP). O efeito da interação desse extrato com o feromônio foi avaliado em machos. A percepção eletrofisiológica de machos e fêmeas aos diferentes extratos de planta não variou com a condição alimentar. Para ambos os sexos, o EBP gerou as maiores respostas eletrofisiológicas. O comportamento quimiotáxico ao EPB tendeu a ser maior em fêmeas copuladas. Na percepção eletrofisiológica, houve efeito aditivo entre o atraente sexual e o EBP em antenas de machos. No entanto, os resultados comportamentais não corroboraram o observado em eletroantenografia. Os resultados obtidos auxiliam na compreensão da influência dos fatores endógenos na comunicação química entre G. molesta e seus hospedeiros, possibilitando maior adequação e confiabilidade na utilização do controle comportamental com o uso de infoquímicos.

PALAVRAS-CHAVE: Eletroantenografia, olfatometria, mariposa-oriental, controle comportamental, feromônio.

\begin{abstract}
INFLUENCE OF ENDOGENOUS FACTORS ON THE ELECTROPHYSILOGICAL AND BEHAVIORALPERCEPTIONOFHOSTPLANT VOLATILESBY GRAPHOLITAMOLESTA(BUSCK) (LEPIDOPTERA: TORTRICIDAE). The oriental fruit moth, Grapholita molesta (Lep.: Tortricidae), stands out as one of the most important pests in Rosaceae orchards in Brazil. During feeding, caterpillars bore into shoots, branches and fruits, impairing the commercial production. The present work was aimed to study the influence of the host plant volatiles and the interaction of these with synthetic sex pheromone in the chemical perception and in the chemotactic behavior (olfatometry) of this species. The electroantennographic perception of both sexes of G. molesta that had been fed or not fed extracts of shoots and ripe and unripe fruits of apple (Malus domestica, Gala cv.) and peach (Prunus persicae, Chiripá cv.) trees was observed. The endogenous factors evaluated were the age, the mating status and the feeding in response to peach-tree shoots extract (PTSE). The effect of the extract interaction with pheromone was evaluated in males. The electrophysiological perception of the plant volatiles by males and females did not vary with feeding status. For both sexes, PTSE elicited the stronger electrophysiological responses. The chemotactical behavior in the presence of PTSE showed a tendency to be higher in mated females. An additive effect was noticed between the sexual attractant and the PTSE in the males' electrophysiological perception. However, the behavioral results did not corroborate with those obtained in the electroantennography. The results obtained aid in the understanding of the interference of endogenous factors in the chemical communication among G. molest and host plants, improving the adaptation and reliability of infochemicals in the behavioral control of the oriental fruit moth.
\end{abstract}

KEYWORDS: Eletroantennography, olfactometry, oriental fruit moth, behavioral control, pheromone.

*Programa de Pós-graduação em Fitotecnia, Faculdade de Agronomia, Universidade Federal do Rio Grande do Sul 


\section{INTRODUÇÃO}

A mariposa-oriental, Grapholita molesta (Busck) (Lepidoptera: Tortricidae), é uma das principais pragas dasculturas da macieirae do pessegueirono Brasil,cujos danos são decorrentes da alimentação e das galerias das lagartas nos brotos, ramos novos e frutos (SALLES, 2001).

A utilização do feromônio sexual sintético é uma prática adotada para monitoramento e controle comportamental da mariposa-oriental (BоTTON et al., 2005; MONTEIRO et al., 2008).

Noentanto,paraqueousodeferomôniossejaaprimorado e ampliado, são necessários estudos que busquem maior compreensão a respeito da biodinâmica desses atraentesedepossíveisefeitosaditivosousinérgicoscom voláteis de plantas. Trabalhos dessa natureza podem elucidar as influências dos fatores fenológicos das plantas hospedeiras na ocorrência, distribuição e flutuação populacional de insetos (MetCAlF; MetCALF, 1992).

As plantas produzem diferentes substâncias químicas que atuam na comunicação inseto-planta (Metcalf; Metcalf, 1992). Órgãos distintos de um mesmohospedeiro podem liberar diferentes voláteis secundários, os quais podem variar com o estádio fenológico da planta (Horvat; CHAPMAN, 1990). O reconhecimento das plantas hospedeiras por ambos os sexos possibilita o encontro de parceiros para cópula e sítios de oviposição, conforme o observado em Cydia pomonella L. (Lep.: Tortricidae) (MASANTEROCA et al., 2002; ANSEBo et al., 2004). A condição alimentar e o status de cópula, este especialmente em fêmeas, podem interferir na capacidade perceptiva aos cairomônios liberados pelos hospedeiros. Para Lobesia botrana (Denis e Schiffermüller) (Lep.: Tortricidae) o status de cópula foi relevantena percepção aos odores de planta (MASANTE-RocA et al., 2002).

Em experimentos eletroantenográficos e comportamentais, foi constatado que compostos secundários de plantas apresentam ação sinérgica e/ ou aditiva com feromônios na atratividade de machos (Stelinski etal.,2003; Deng et al., 2004; YAng etal., 2004).

Neste contexto, o trabalho objetivou estudar a influência de voláteis de plantas hospedeiras e a interação destes com o feromônio sexual sintético na percepção eletrofisiológica (EAG) e no comportamento quimiotáxico (olfatometria) de G. molesta, visando à compreensão da influência dos fatores endógenos na comunicaçãoquímica entreoinsetoeseushospedeiros, nosentidodeaprimoraros conhecimentos a respeitoda biodinâmicadeferomôniosecairomôniosnestaespécie.

\section{MATERIALEMÉTODOS}

Os insetos utilizados foram provenientes de uma criação de manutenção conduzida conforme ARIOLIet al (2007), em sala climatizada $\left(25 \pm 1^{\circ} \mathrm{C} ; 60 \pm 10 \%\right.$ U.R.; fotofase de 16 horas e luminância igual a 1,46 klx), onde os adultos eram mantidos em gaiolas formadas por garrafa PET, com solução de mel a 15 \% e Nipagin ${ }^{\circledR}$ a $0,15 \%$ disponível para alimentação em algodão embebido. As fêmeas realizavam as posturas na parede interna da garrafa e esta era recortada após 2 dias e seus pedaços, contendo os ovos de G. molesta, eram colocados sobre dieta artificial à base de maçã seca e farinha de milho para desenvolvimento das lagartas. Para o estabelecimento da criação, foram coletados insetos em pomares de pessegueiro, em Bento Gonçalves, RS, mantidos por muitas gerações em dieta artificial até a realização dos experimentos.

Partes vegetais (brotos, frutos verdes e frutos maduros) depessegueiro Prunuspersicae (cultivarChiripá) e de macieira, Malus domestica, (cultivar Gala) foram coletadas em pomares orgânicos localizados em Pinto Bandeira, RS ( $29^{\circ} 06^{\prime} \mathrm{S}$ e $\left.51^{\circ} 27^{\prime} \mathrm{W}\right)$, em outubro/ 2006 ejaneiro/2007e em Antônio Prado, RS (2851'30" Se $51^{\circ} 16^{\prime} 58^{\prime \prime} \mathrm{W}$ ), em fevereiro/2007, respectivamente. Em ambos os pomares não foi utilizado tratamento químico para controle de pragas ou doenças.

Foramelaborados extratoscombrotos, frutosverdes efrutosmaduros depessegueiro(EBP,EPV eEPM)ede macieira (EBM, EMV e EMM), obtidos por meio da técnica de refluxo, onde os materiais foram submetidos à extração sólido-líquido com o solvente etanol (Vetec, 99\% depureza). As partesvegetaisforam picadasemum liquidificadore, então, $50 \mathrm{~g}$ de amostra foram pesadase transferidas para um balão volumétrico de $100 \mathrm{~mL}$. Foram adicionados $20 \mathrm{mLdesolventesobreasamostras}$ eestas foram submetidas a refluxo por um período de 4 horas. Os extratos obtidos repousaram por 1 hora e, então, foram filtrados. As extraçõesforam realizadasno período de 24 a 48 horas após a coleta do material. Os extratos foram armazenados sob refrigeração.

As técnicas eletroantenográficas utilizadas são similaresàs deTRIMBLE;MARSHALL (2007). Machosefêmeas de G. molesta com até um dia de idade foram individualizadosem recipientes plásticos $(500 \mathrm{~mL})$ emantidosaté que atingissema idadeestabelecida para os bioensaios, recebendo solução demel a $15 \%$ eNipagin $\AA$ a $0,15 \%$ ou apenas água. A resposta eletroantenográfica foi capturada, amplificada e processada com um controlador de aquisição de dados (IDAC-4, Syntech ${ }^{\circledR}$ ) e, posteriormente, registrada por software (EAG2000, Syntech $\left.{ }^{\circledR}\right)$.

Uma alíquota de feromônio ou de extrato de planta era aplicada sobre papel filtro $(1,5 \times 2,5 \mathrm{~cm}) \mathrm{com}$ dobras em forma de sanfona. O estímulo controle foi papel filtro impregnado com o solvente no mesmo volume da amostra testada, podendo ser etanol, hexano ou ambos.

As antenas foram submetidas a pulsos de ar, gerados por um controlador de fluxo (CS-02, 
Syntech $\left.{ }^{\circledR}\right)$, num volume de $2,5 \mathrm{~mL} / 0,5 \mathrm{~s}$, com os diferentes tratamentos testados. Estipulou-seo tempo de um minuto entre sucessivos estímulos Os papéis filtro contendo os extratos e o feromônio foram renovados a cada seis antenas testadas, expondo-se os tratamentos de forma aleatória.

Foi utilizado o solvente etanol como controle nos testes relativos à percepção olfativa aos voláteis de planta e o hexano quando o feromônio era o estímulo. Todos os extratos vegetais foram testados em insetos virgens de ambos os sexos (separados sob estereomicroscópio, na fase pupal, com base no número de segmentos abdominais aparentes), alimentados e não-alimentados, com três dias de idade.

Posteriormente, avaliou-se a resposta de fêmeas alimentadas, virgens e não virgens de 4,7,10 e 13 dias deidade, somente ao extrato de broto de pessegueiro (EBP), devidoà superioridade das respostas a este no experimento anterior. As fêmeas não virgens foram pareadas logo após a emergência com um macho de mesma idadee mantidas desta forma atéo dia anterior ao experimento. Após a realização do bioensaio, as bursas copulatórias foram retiradas do abdômen das fêmeas com auxílio de estilete e examinadas sob estéreomicroscópio, para certificação da cópula.

Para avaliar a interação de voláteis de partes das plantas hospedeiras e do feromônio sexual sintético na resposta eletrofisiológica de machos de G. molesta, indivíduos recém-emergidos e virgens foram individualizados, alimentados e mantidos nestas condições por três dias, quando foram realizados os testes.

Os atraentes utilizados nestes bioensaios foram os solventes controle (etanol para os extratos vegetais e hexanoparaoferomônio), testadosisolados $(5 \mu \mathrm{L})$ ouem mistura ( $5 \mu \mathrm{L}$ de cada), bem como o feromônio e o EBP isoladamente $(5 \mu \mathrm{L})$. Quandoutilizados em conjunto, $\mathrm{o}$ volume da mistura feromonal (MF) foi sempre $5 \mu \mathrm{L}$, enquantooEBP foi empregado em dois volumes, $5 \mu \mathrm{Le}$ $7,5 \mu \mathrm{L}$. A primeira proporção $(5 \mu \mathrm{LMF}+5 \mu \mathrm{LEBP})$ visou compararestas respostascomas dossolventes, emEAG. A segunda ( $5 \mu \mathrm{L} \mathrm{MF}+7,5 \mu \mathrm{LEBP})$ objetivou comparar as respostas de percepção eletroantenográficas com as dos testes comporta-mentais, nos quais os volumes utilizadosforam de $10 \mu \mathrm{L}(\mathrm{MF})+15 \mu \mathrm{L}(\mathrm{EBP})$. Osvolumes maiores usadosnos testes comportamentais foramestabelecidos após experimento piloto.

Nos experimentos comportamentais com machos efêmeas, utilizou-se somente oEBP, selecionadocom base nos experimentos eletroantenográficos. O comportamento de adultos foi observado em olfatômetro "Y" de vidro de dupla escolha, com diâmetro de $4 \mathrm{~cm}$, arena inicial de $22 \mathrm{~cm}$, bifurcada em dois "braços" de $24 \mathrm{~cm}$ cada, conforme GöKçE et al. (2005), em capela com sistema de exaustão de ar, em sala climatizada $\left(25 \pm 1^{\circ} \mathrm{Ce} 60 \pm 10 \%\right.$ U.R.). O fluxo de ar, previamente filtrado com carvão ativo, foi conduzido para dentro do sistema com um propulsor conectado a um fluxímetro, a uma taxa de $0,79 \mathrm{~L} / \mathrm{min}$. Testes com machos foram conduzidos na fotofase sob luz incandescente $(60 \mathrm{~W}$, luminância igual a $0,518 \mathrm{klx})$ e os com fêmeas, na escotofase, com lâmpada vermelha (70 W, luminância igual a 135 lx).

Ocomportamento quimiotáxico de machos, frente à interação EBPe feromônio, foi observadoem indivíduos virgens, de cinco dias de idade e alimentados com solução de mel a $15 \%$ e Nipagin $®$ a $0,15 \%$. Nos testes em que se avaliou somenteEBP, utilizou-se um volume de $15 \mu \mathrm{Le}$, naqueles em quea interaçãoe extrato eferomônio foi verificada, $15 \mathrm{e} 10 \mu \mathrm{L}$, respectivamente, totalizando $25 \mu \mathrm{L}$. Como controle, empregou-se os solventes etanol ehexano, isoladamente ou em mistura, em volumes iguais aos dos extratos.

Considerou-se resposta positiva quando os insetos percorreram, pelomenos, $4 \mathrm{~cm}$ dentrodosbraçoscontendootratamentotesteepermaneceramnestaárea por, no mínimo,1 min,negativa, quandopercorressema mesma distância eficassem omesmotemponobraçocontendo o controle, e ausência de resposta, quando permaneceram apenas no braço principal do olfatômetro. Foram realizadas, no mínimo, 30 repetições para cada tratamento e cada inseto foi observado por 10 minutos.

Nos dois experimentos(EAGeolfatometria) foram calculadas médias, erros padrões e variâncias. Os resultados do EAG foram comparados por ANOVA, com $95 \%$ de confiabilidadee os dados de olfatometria pelo Teste $t$ ou Exato de Fischer, com 95\% de confiabilidade. Nas análises foram utilizandos os softwares Bioestat ${ }^{\circledR} 4.0$ e SPSS ${ }^{\circledR}$ for Windows 13.0.

\section{RESULTADOSEDISCUSSÃO}

A resposta eletrofisiológica de fêmeas de G. molesta foi significativamente maior para o $\operatorname{EBP}(23,12 \pm 2,264$ $\mathrm{mV})$, seguido do EBM $(14,34 \pm 1,198 \mathrm{mV})(\mathrm{P}<0,01)$. Não houve diferença entre os demais tratamentos e o etanol ( $\mathrm{P}=0$,364) ( $\mathrm{F}=18,395)$ (Fig.1) Resultado semelhante foi encontrado para machos, sendo as respostas eletroantenográficas ao $\mathrm{EBP}(35,24 \pm 3,395 \mathrm{mV})$ e EBM $(26,06 \pm 2,913 \mathrm{mV})$ diferentes do controle $(\mathrm{P}<$ $0,01)$ eos demais tratamentos, estatisticamenteiguais ao etanol $(P=0,250)(F=6,938)$ (Fig. 2$)$. As respostas eletrofisiológicas de machos a todos os tratamentos foram superiores às de fêmeas $(\mathrm{P}<0,01)$. No entanto, não é possível associar este resultado a uma maior bioatividade desses extratos em antenas de machos, uma vez que as respostas obtidas com o tratamento controle (etanol) também foram significativamente superiores neste sexo. Assim, as diferenças nas médias de despolarização $(\mathrm{mV})$ entreos sexos podem estar relacionadas à maior sensibilidade das antenas de machos ao solvente etanol. 
A percepção química aos voláteis de planta, de modo geral, é realizada por machos e fêmeas. No entanto, supõe-se que, em G. molesta, os sexos utilizem esta percepção para desempenhar, principalmente, duas funções biológicas distintas. Para machos e fêmeas, os voláteis secundários teriam o objetivo de orientar os parceiros sexuais para plantas hospedeiras preferidase, posteriormente, sítios de cópula. Já as fêmeas, utilizariam este recurso para localizar sítios de oviposição relacionados a hospedeiros palatáveis para sua prole (METCALF; MetCALF, 1992).

A seleção do hospedeiro por insetos fitófagos é mediada por uma série complexa defatores evolutivos que envolvem mecanismos de atratividade e defesa. Segundo MetCalf; Metcalf (1992), órgãos distintos de uma mesma planta, como flores, folhas e frutos, em diferentes estádios de maturação, podem apresentar variações na produção e liberação de substâncias químicas voláteis. Em Cydia pomonella já foram constatados diferentes níveis de atratividade aos voláteis emitidos por distintas partes da planta hospedeira e estádios de desenvolvimento (HORvat; CHAPMAN, 1990; CASADO et al., 2006). PIÑERO; DORN (2007), avaliando o efeito sinérgico de dez compostos químicos sintéticos, presentes em broto de pessegueiro, na atratividade de fêmeas não virgens de Cydia (= Grapholita) molesta , em olfatômetro de dupla escolha, concluíram quea mistura de cinco substâncias foi tão atrativa quanto o broto depessegueiro in natura, sendoa mistura composta por (Z)-3-acetato dehexenila,(Z)-3-hexenol, benzaldeído, (E)-2-hexanal e benzonitrila. Segundo os autores, a presença de benzaldeído e benzonitrila na mistura é fundamental na atratividade de fêmeas. No entanto, quando estes foram testados de forma isolada, não desencadearam comportamentodeatratividadeou de repelência. Os autores observaram que o benzaldeído foi o composto majoritário nas amostras de broto de pessegueiro,nãosendoobservadoemextratos dofruto. Desta forma, assim como foi observado por PIÑERO; DORN (2007), a presença do benzaldeído no extrato da planta parece ser importante para o aumento da percepção aos voláteis do pessegueiro.

Em macieira, os compostos predominantes são o (Z)-3-acetato de hexenila, (Z)-3-hexenol, ( $E, E)-\alpha$ farneseno, acetato dehexenila, hexanoato dehexenila e germacreno (ANSEBO et al., 2004; CASADO et al., 2006). Entretanto, não foi feita análise cromatográfica comparativa entre os extratos de macieira utilizados nessebioensaio parajustificar quimicamente o resultado superior apresentado pelo broto de macieira.

Apesardas variações entreotipoea concentraçãode voláteis em determinadas partes e/ou fases de desenvolvimentodaplanta,NATALEetal.(2003), emolfatômetro, nãoencontraram diferençasnapercepçãoquimiotáxica de fêmeas de C. pomonella a brotos de pessegueiro e de macieira. Em C. molesta (= G. molesta) foi observado comportamentosemelhante, ou seja, tantovoláteisemitidos por pêssego como por maçã, verdes e maduros, foramatrativosafêmeasnãovirgens(NATALEetal.,2004).

As médias das respostas eletroantenográficas periféricas de fêmeas virgens e copuladas de 4,7 e 13 dias de idade não diferiram significativamente entre si quando estimuladas com o $\mathrm{EBP}(\mathrm{P}>0,05, \mathrm{~F}=4,076)$. No entanto, fêmeas copuladas de sete a dez dias foram mais responsivas a esta fonte de estímulo do que as fêmeas virgens $(P<0,05, F=5,423)$ (Tabela 1).

MASANTE-ROCA etal. (2002) concluíram que as respostas de machos e fêmeas de Lobesia botrana (Denis e Schiffermüller)(Lep.:Tortricidae) a 13voláteis presentes em videiras não diferiram, independente do sexo e do status decópula. Entretanto, constataram uma tendência derespostasmaioresemfêmeascopuladas,emdetrimento das de machos copulados e de fêmeas virgens.

Esta tendência também foi observada neste trabalho em bioensaios comportamentais, no qual o status de cópula parece ter influenciado a percepção de fêmeas aos voláteis presentes neste extrato, já que sete fêmeas copuladas foram atraídas pelos voláteis do broto de pessegueiro, em contraste com apenas uma virgem(Fig.3). Resultadosemelhante, emolfatometria, foi observado para C. molesta por NATALE et al. (2004), ondefêmeas virgens não responderam aos voláteis de pêssego emaçã, verdese maduros, enquanto ascopuladas foram atraídas por todos, não diferindo no comportamento quimiotáxico, independente da fonte de estímulo. Em túnel-de-vento, Y ANet al (1999) constataram que fêmeas virgensecopuladas deC. pomonella foram mais ativas na presença de voláteis de maçã do que na ausência destes, no entanto, somente as copuladas alçaram voo em direção à fonte de odor.

Tabela 1 - Médias das respostas eletrofisiológicas ( \pm erro padrão), em milivolts (mV), de fêmeas virgens e não virgens de Grapholita molesta de diferentes idades aos voláteis emitidos pelo extrato etanólico de broto de pessegueiro $(\mathrm{n}=10)$.

\begin{tabular}{lrrrr}
\hline Status de cópula & \multicolumn{4}{c}{ Idade (dias) } \\
\cline { 2 - 5 } & \multicolumn{1}{c}{4} & \multicolumn{1}{c}{10} & 13 \\
\hline Virgens & $9,60 \pm 2,209 \mathrm{Ba}$ & $13,95 \pm 1,942 \mathrm{Aa}$ & $12,35 \pm 1,921 \mathrm{ABb}$ & $5,35 \pm 2,222 \mathrm{Ba}$ \\
Não virgens & $21,95 \pm 2,523 \mathrm{Ba}$ & $31,20 \pm 3,963 \mathrm{Aa}$ & $29,80 \pm 3,979 \mathrm{Aa}$ & $18,35 \pm 4,519 \mathrm{Ba}$
\end{tabular}

Médias seguidas de letras distintas maiúsculas, nas linhas, e minúsculas, nas colunas, diferem entre si $(P<0,05)$. 


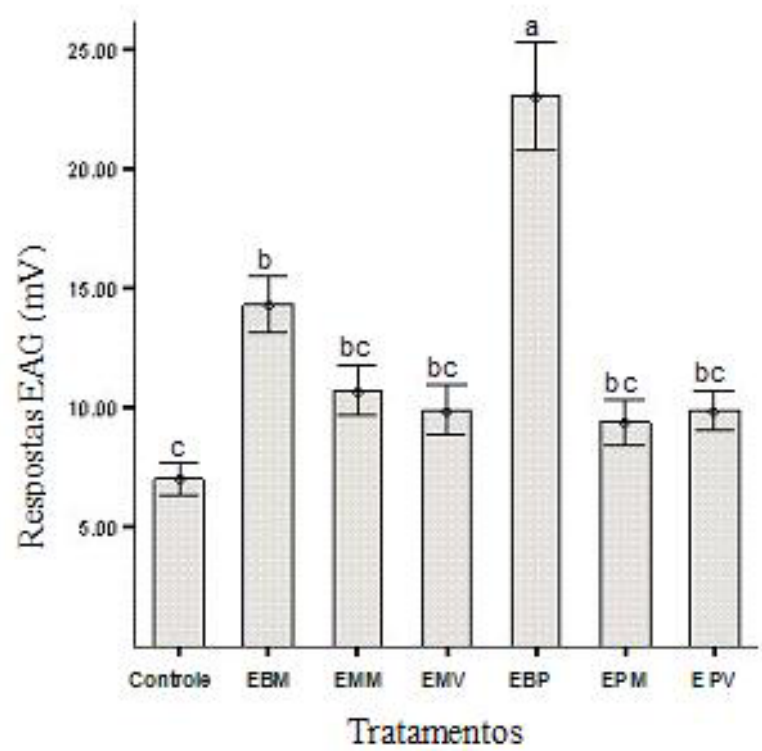

Fig. 1 - Médias das respostas eletrofisiológicos $( \pm$ erro padrão), em milivolts ( $\mathrm{mV}$ ), de fêmeas de Grapholita molesta ( $n=15)$ a voláteis de diferentes extratos etanólicos de plantas hospedeiras: $\mathrm{EBM}=$ broto de macieira; $\mathrm{EMM}=$ maça madura; EMV = maça verde; $\mathrm{EBP}$ = broto de pessegueiro; EPM pêssego maduro; EPV = pêssego verde. Barras seguidas de letras distintas diferem entre si $(\mathrm{P}<0,05)$.

As maiores respostas quimiotáxicas das fêmeas copuladas, em bioensaios comportamentais, corroboram a ideia de MetCalf; Metcalf (1992), de que a maior sensibilidade odorífera aos voláteis da planta hospedeira decorre da necessidade de orientação rápida e seletiva para melhores sítios de oviposição, promovendo a sobrevivência da prole.

As respostas defêmeas virgens desetedias deidade foram significativamentemaiores queas dequatroeas de13dias $(\mathrm{P}<0,05)$, nãodiferindoestatisticamentedas de dez dias $(P=0,0631)(F=3,747)$. Fêmeas copuladas de dez dias desencadearam maiores respostas eletrofisiológicas, diferindo estatisticamente das de quatro $(\mathrm{P}<0,05)$ e 13 dias $(\mathrm{P}<0,01)$ eigualando com as de sete dias $(\mathrm{P}=0,6305)(\mathrm{F}=5,554)$ (Tabela 1).

Assim, não somente o status de cópula influencia a percepção de fêmeas aos voláteis da planta hospedeira; a idade pode interferir na resposta eletroantenográfica. A maior percepção foi observada na faixa de sete a dez dias de idade, no entanto, fêmeas copuladas de dez dias foram mais responsivas queas virgens. $\mathrm{O}$ fato das fêmeas de $G$. molesta serem mais perceptivas nas idades intermediárias pode estar relacionado a ser este o período de maior atividade reprodutiva, em busca de sítios de cópula ou oviposição. A cópula, nas fêmeas de dez dias, parece desencadear mecanismos biossintéticos que promovem uma otimização da capacidade perceptiva na

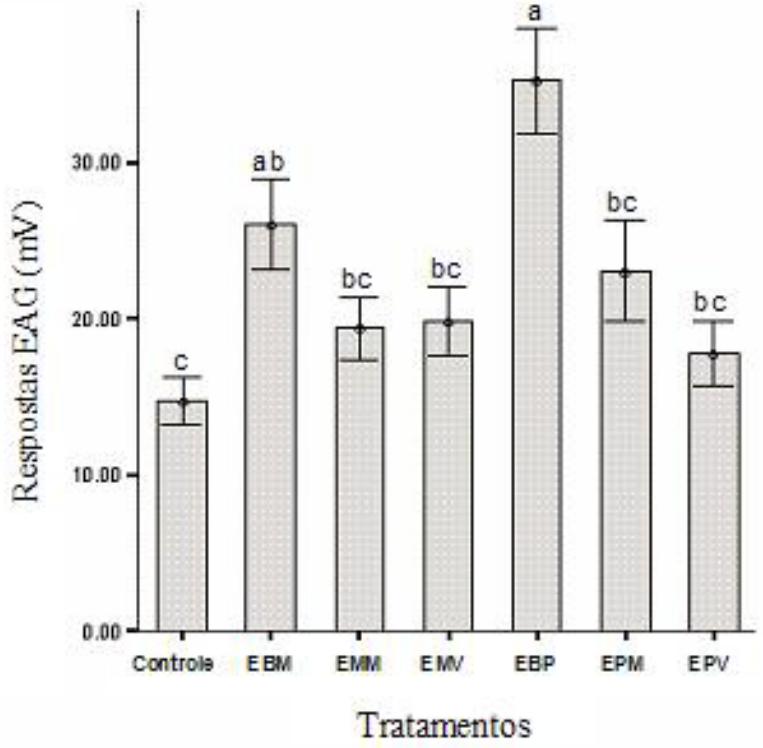

Fig. 2 - Médias das respostas eletrofisiológicos $( \pm$ erro padrão), em milivolts ( $\mathrm{mV}$ ), de machos de Grapholita molesta ( $\mathrm{n}=15)$ a voláteis de diferentes extratos etanólicos de plantas hospedeiras: $\mathrm{EBM}=$ broto de macieira; $\mathrm{EMM}=$ maça madura; $\mathrm{EMV}=$ maça verde; $\mathrm{EBP}$ = broto de pessegueiro; EPM pêssego maduro; EPV = pêssego verde. Barras seguidas de letras distintas diferem entre si $(\mathrm{P}<0,05)$.

identificação e localização do hospedeiro para oviposição.

Considerando-se que a longevidade média de fêmeas é de 16,28 dias (Alfonso; MARín, 2004), pode-se supor que no período de sete a dez dias a sensibilidade olfativa relacionada à localização do hospedeiro esteja no ápice, já que nesta fase a maioria dos indivíduos já copulou.

A percepção eletrofisiológica de fêmeas e machos aos voláteis dos hospedeiros, em função da condição alimentar, não apresentou diferenças significativas (P > 0,05). Segundo DiCKENS (1997), os açúcares não participam diretamente das rotas biossintéticas de substâncias envolvidas na percepção química, sendo as proteínas, aminas e hormônios, os principais responsáveis por esta função.

Observou-se efeito aditivo, porém não significativo(Fig. 2), nas respostas eletrofisiológicas deantenas de machos de G. molesta quando elas foram estimuladas com a mistura do feromônio sexual e o EBP. Esta interação só foi significativamente diferente do feromônio, quando ovolume de extrato adicionado a este foi de $7,5 \mu \mathrm{L}(47,93 \mathrm{mV} \pm 2,896)(\mathrm{P}<0,01, \mathrm{~F}=$ $27,234)$.

Os bioensaios comportamentais não corroboraram o observado nos testes com EAG, ou seja, não foram constatados efeitos aditivos e/ou sinérgicos entre oferomônio e o $\operatorname{EBP}(\mathrm{P}=0,281, \mathrm{~F}=8,245)$ (Fig. 4). 


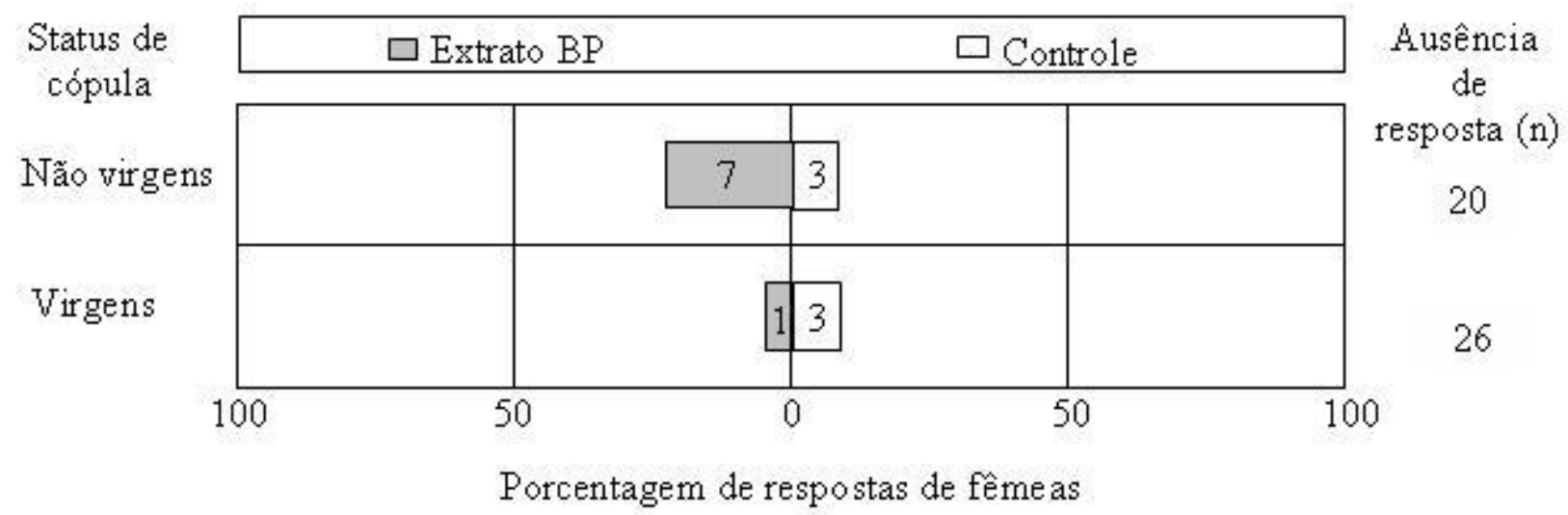

Fig. 3 - Proporções e porcentagens de respostas comportamentais de fêmeas de Grapholita molesta, não virgens $(\mathrm{n}=30)$ e virgens $(n=30)$, ao extrato etanólico de broto de pessegueiro (EBP) em olfatômetro "Y". Números dentro das barras referem-se ao número de indivíduos que respoderam aos tratamentos.

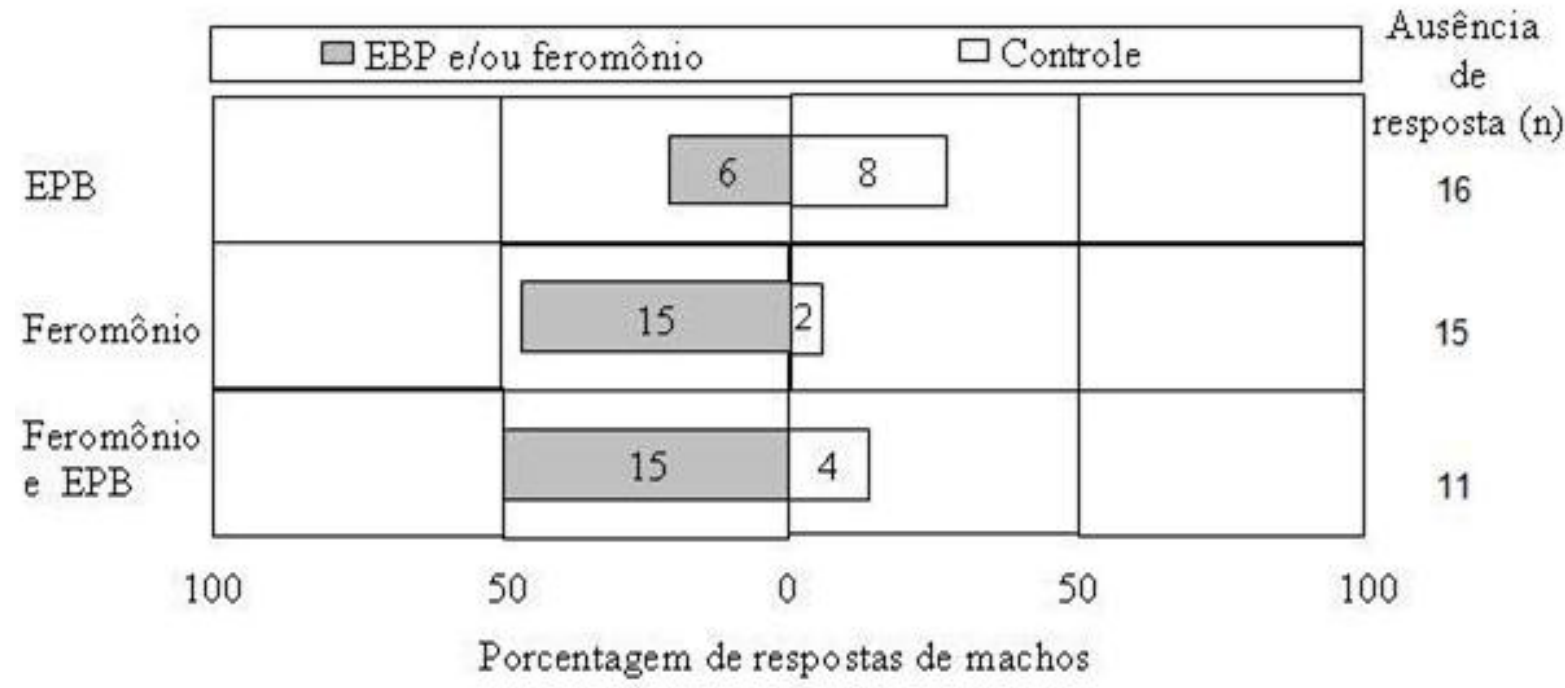

Fig. 4 - Proporções e porcentagens de respostas comportamentais de Machos de Grapholita molesta, ao extrato etanólico de broto de pessegueiro (EBP) $(n=30)$, ao fenômeno sexual $(n=30)$ e a interação destes dois $(n=30)$ em olfatômetro "Y". Números dentro das barras referem-se ao número de indivíduos que respoderam aos tratamentos.

Os resultados evidenciaram que a percepção das antenas de machos de G. molesta aumenta com a adição de voláteis de planta ao feromônio sintético. YANG et al. (2004) contataram que, entre diversos voláteis de maçã, apenas farneseno, em altas concentrações, foi capaz de estimular o comportamento de vôo de C. pomonella. Estes autores observaram que há um efeito sinérgico, em túnel-de-vento, entre o feromônio sexual de C. pomonella (E,E-8,10-dodecadienol), em mistura com três voláteis secundários de maçã:linalol, (E)-b-farneseno, (Z)-3-hexenol. Deacordocom Light et al. (1993), a captura de machos de C. pomonella, em bioensaios de campo, foi incrementada com a adição de voláteis de plantas às armadilhas de feromônio. OcHIENG etal. (2002) observaram que neurônios receptores deestímulos químicos em antenas de machos de Helicoverpa zea (Boddie) (Lep.: Noctuidae) são mais responsivos quando estimulados com a mistura de feromônio sexual evoláteis deplanta, tais comolinalol e (Z)-3-hexenol, do que quando submetidos a estes infoquímicos isoladamente.

Em termos co-evolutivos, a interação entre voláteis deplanta eferomônio de insetos fitófagos pode representar um importante indicador químico utilizado por estes para discriminar suas plantas hospedeiras e ali se agregarem para atividades de cópula, oviposição e de desenvolvimento das formas imaturas. YANG et al. (2004) verificaram que alguns voláteis demaçã, como o (E)-b-farneseno, podem amplificar a atratividade de machos de C. pomonella quando a concentração de feromônio no ambiente é baixa.

Sendo assim, interação inseto-planta pode ser um fator relevante para a sobrevivência das espécies. WitzGall et al. (1991) observaram que fêmeas de 
Spilonata ocellana (Denis \& Schiffermüller) e S. laricana (Heinemann) (Lep.: Tortricidae) compartilham o mesmo feromônio sexual. No entanto, a captura de machos de cada uma das espécies em armadilhas de campoédiretamente influenciada pelo tipo dehospedeiro onde a armadilha écolocada, ou seja, machos de S. ocellana são atraídos em armadilhas colocadas em macieiras, enquanto que os de $S$. laricana, naquelas situadas em pinheiro.

Os resultados permitem concluir que o extrato etanólico de broto de pessegueiro (EBP) desencadeia as maiores respostas eletroanteonográficas em ambos os sexos de G. molesta, independentemente da condição alimentar e do status de cópula, em fêmeas. A percepção eletroantenográfica de fêmeas ao EBP varia com a idade. Os voláteis debroto de pessegueiro provocam um feito aditivo na percepção eletroantenográfica de machos ao feromônio e a interação EBP-feromônio não interfere no comportamento quimiotáxico de machos.

O fato de ambos os sexos serem mais seletivos ao EBP e este ter efeito aditivo na percepção eletroantenográfica, quando em combinação com o feromônio, sugere que substâncias bioativas presentes neste extrato, como o benzaldeído, descrito por HoRvat; Chapman (1990) como um dos principais compostos aromáticos presentes no pessegueiro, possam ser empregadas em armadilhas de campo, possibilitando a captura de ambos os sexos, o que potencializaria o uso desta ferramenta no controle de G. molesta em agroecossistemas.

\section{AGRADECIMENTOS}

Ao Dr. Luiz A.M. Fontoura e Fabrício Naciuk, do Laboratório de Química da CIENTEC - Fundação de Ciência e Tecnologia (Cachoeirinha, RS), pela elaboração dos extratos de planta e diluições feromonais; ao Dr. Marcos Botton, da EMBRAPA Uva e Vinho (Bento Gonçalves), pelo fornecimento de G. molesta:à Isca Tecnologias Ltda., pelo feromônio sintético e ao Conselho Nacional de Desenvolvimento Científico e Tecnológico (CNPq) pela concessão de bolsas ao primeiro, terceiro e quarto autores.

\section{REFERÊNCIAS}

ALFONSO, A. M.; MARÍN, M. S. Grapholita molesta em condiciones de laboratório: evaluación de la relación de sexos. Revista de la Facultad de Ciencias Exactas y Naturales, v.36, n.2, p.23-29, 2004.

ANSEBO, L.; CORACINI, M.D.A.; BENGTSSON, M.; LIBLIKAS, I.; RAMÍREZ, M.; BORG-KARLSON, A.K.; TASIN, M.; WITZGALL, P. Antennal and behavioural response of codling moth Cydia pomonella to plant volatiles. Journal of Applied Entomology, v.128, n.7, p.488493, 2004.

ARIOLI, C. J.; MOLINARI, F.; BOTTON, M.; GARCIA, M. S. Técnica de criação de Grapholita molesta (Busck, 1916) (Lepidoptera: Tortricidae) em laboratório utilizando dieta artificial para a produção de insetos visando estudos de comportamento e controle. Boletim de Pesquisa e Desenvolvimento, Embrapa Uva e Vinho, n.13, 2007.

BOTTON, M. F.; KULCHESKI, V.D.; COLLETTA, C.J.; ARIOLI, C.J.; PASTORI, P.L. Avaliação do uso do feromônio de confundimento no controle de Grapholita molesta (Lepidoptera: Tortricidae) em pomares de pessegueiro. Idesia, v.23, p.43-50, 2005.

CASADO, D.; GEMENO, C.; AVILLA, J.; RIBA, M. Daynight and phenological variation of apple tree volatiles and electroantennogram responses in Cydia pomonella (Lepidoptera : Tortricidae). Environmental Entomology, v.35, n.2, p.258-267, 2006.

DENG, J.Y.; WEI, H.Y., HUANG, Y.P.; DU, J.W Enhancement of attraction to sex pheromones of Spodoptera exigua by volatile compounds produced by host plants. Journal of Chemical Ecology, v.30, p.2037-2045, 2004.

DICKENS, J. Neurobiology of pheromonal signal processing in insects. In: CARDÉ, R.T.; MINKS, A.K. (Ed.) Insect pheromone research: new directions. New York: Chapman \& Hall, 1997. p.210-217.

GÖKÇE, A.; STELINSKI, L.L; WHALON, M.E. Behavioural and electrophysiological responses of leafroller moths to selected plants extracts. Environmental Entomology, v.34, p.1426-1432, 2005.

HORVAT, R.J.; CHAPMAN, G.W. Comparison of volatile compounds from peach fruit and leaves (cv. Monroe) during maturation. Journal of Agricultural and Food Chemistry, v.38, p.1442-1444, 1990.

LIGHT, D.M.; FLATH, R.A.; BUTTERY, R.G.; ZALOM, F.G.; RICE, R.E.; DICKENS, J.C.; JANG, E.B. Host-plant green-leaf volatiles synergize the synthetic sex pheromones of the corn earworm and codling moth (Lepidoptera). Chemoecology, v.4, p.145-152, 1993.

MASANTE-ROCA, I.; GADENNE, C.; ANTON, S. Plant odour processing in the antennal lobe of male and female grapevine moths, Lobesiana botrana (Lepidoptera: Tortricidae). Journal of Insect Physiology, v.48, p.1111-1121, 2002.

METCALF, R.L.; METCALF, E.R. Plant kairomones in insect ecology and control: contemporary topics in entomology 1. London: Chapman \& Hall, 1992. 168p. 
MONTEIRO, L.B.; SOUZA, A.; BELLI, E.L. Confusão sexual para o controle de Grapholita molesta (Lepidoptera: Tortricidae), em pomares de macieira, em Fraiburgo (SC) Brasil. Bragantia, v.67, p.191-196, 2008.

NATALE, D.; MATTIACCI, L.; HERN, A.; PASQUALINI, E.; DORN, S. Response of female Cydia molesta (Lepidoptera: Tortricidae) to plant derived volatiles. Bulletin of Entomological Research, v.93, p.335-342, 2003.

NATALE, D.; MATTIACCI, L.; HERN, A.; PASQUALINI, E.; DORN, S. Apple and peach fruit volatiles and the apple constituent butyl hexanoate attract female oriental fruit moth, Cydia molesta, in the laboratory. Journal of Applied Entomology, v.128, p.22-27, 2004.

OCHIENG, S.A.; PARK, K.C.; BAKER, T.C. Host plant volatiles synergize responses of sex pheromone-specific olfactory receptor neurons in male Helicoverpa zea. Journal of Comparative Physiology, v.188, p.325-333, 2002.

PIÑERO, J.C.; DORN, S. Synergism between aromatic compounds and green leaf volatiles derived from the host plant underlies female attraction in the oriental fruit moth. Entomologia Experimentalis et Applicata, v.125, n.2, p.185-194, 2007.

SALLES, L.A. Mariposa-oriental, Grapholita molesta (Lepidoptera: Tortricidae). In: VILELLA, E.F.; ZUCCHI, R.A.; CANTOR, F. (Ed.). Histórico e impacto das pragas introduzidas no Brasil. Ribeirão Preto: Holos, 2001. p.42-45.
STELINSKI, L.L; MILLER, J.R.; RESSA, N.E.; GUT, L.G. Increased EAG responses of tortricid moths after prolonged exposure to plant volatiles: Evidence for octopamine-mediated sensitization. Journal of Insect Physiology, v.49, p.845-856, 2003.

TRIMBLE, R.M.; MARSHALL, D.B. Quantitative method for pheromone delivery in studies of sensory of moth antennae. Physiological Entomology, v.32, p.388393, 2007.

YAN, F.; BENGSTON, M.; WITZGALL, P. Behavioral Response of Female Codling Moths, Cydia pomonella, to Apple Volatiles. Journal of Chemical Ecology, v.25, n.6, p.1343-1351, 1999.

YANG, Z.; BENGSTON, M.; WITZGALL, P. Host plant volatiles synergize response to sex pheromone en codling moth, Cydia pomonella. Journal of Chemical Ecology. v.30, n.3, p.619-629, 2004.

WITZGALL, P.B.; BENGTSSON, M.; BUSER, H.R.; CHAMBON, P.J.; PRIESNER, E.; WILDBOLZ, T.; ARN, H. Sex pheromones of Spilonata ocellana and Spilonata laricana. Entomologia Experimentalis et Applicata, v.60, p.219-223, 1991.

Recebido em 5/6/08

Aceito em 24/7/09 